\title{
ANÁLISIS MULTITEMPORAL Y MULTIFRACTAL DE LA DEGLACIACIÓN DE LA CORDILLERA PARÓN EN LOS ANDES DE PERÚ
}

\section{MULTITEMPORAL AND MULTIFRACTAL ANALYSIS OF THE PARON RANGE DEGLACIATION IN THE ANDES OF PERÚ}

\author{
Gilberto Medina ${ }^{1}$ y Abel Mejía ${ }^{2}$
}

\begin{abstract}
Resumen
La pérdida del volumen glaciar de los nevados tropicales de nuestro país puede ser considerada como un indicador del cambio climático a nivel global. Según afirma Urrutia \& Vuille (2009) para el año 2050, todos los glaciares ubicados por debajo de los $5500 \mathrm{msnm}$ desaparecerían. Una de las técnicas científicas empleadas para describir el sistema poroso del suelo (Montero, 2003) y para analizar las concentraciones de ulexite en minas de sal (Posadas et al., 2005), es el uso de multifractales, que ha sido escasamente aplicado al sistema glaciar. El objetivo de este trabajo es cuantificar el volumen glaciar de la Cordillera Parón en los últimos 24 años empleando tanto técnicas multifractales como el Sistema de Información Geográfica para determinar en qué medida los eventos El Niño y La Niña influyen en su desglaciación. Para diferenciar la masa glaciar de otros elementos como nubes, agua, etc., en las imágenes seleccionadas, se utilizó el NDSI o índice de nieve y también el ratio imagen 4/5 en las imágenes binarizadas. La superficie glaciar de la Cordillera Parón se ha reducido en 18\% en los últimos 24 años (Bates et al., 2008).

Palabras clave: multifractales, dimensión fractal, NDSI, ratio imagen 4/5, box counting, análisis multitemporal.
\end{abstract}

\begin{abstract}
The loss of glacier volume in the tropical glaciers in our country can be regarded as an indicator of global climate change. According to Urrutia \& Vuille (2009), all glaciers below 5500 meters could disappear by the year 2050. One of the scientific techniques used to describe soil pores' system (Montero, 2003) and ulexite concentrations in a salt mine (Posadas et al., 2005) is the use of multifractals, which has been poorly applied in glacier's systems. The focus of this study was to quantify the glacier volume at Parón Range during the last 24 years using multifractal techniques and geographic system information in order to determine to what extent the El Niño and La Niña events influence its deglaciation. To differentiate the glacier mass from other elements such as clouds, water, etc., in the selected images the NDSI or snow index was used as well as the image ratio of $4 / 5$ in the binarized images. The glacier surface at Parón Range has suffered a reduction of $18 \%$ in the last 24 years.
\end{abstract}

Key words: multifractals, fractal dimension, NDSI, ratio image 4/5, box counting, multitemporal analysis.

\section{Introducción.}

Los glaciares andinos son de interés para los científicos, dado que constituyen importantes indicadores del cambio climático, los cuales juegan un importante rol en el manejo del recurso hídrico, actúan como reguladores del régimen hidrológico en casi toda la región andina, $\mathrm{y}$ pueden ser directa $\mathrm{O}$ indirectamente, causa de catástrofes (PNUMA, 2007).

Una de las técnicas disponibles para estudiar elementos que crecen y decrecen en el tiempo, como las nubes, glaciares, etc., es el uso de la técnica multifractal, que ha sido utilizada por Posadas et. al. (2005) como estadística estocástica para analizar concentraciones de ulexite en una mina de sal, a tajo abierto, en Bolivia.

El concepto de multifractales contempla un número infinito de dimensiones fractales; un proceso multifractal se caracteriza por estudiar eventos extremos y más o menos aislados (Posadas et al., 2002).

A pesar de las ventajas conceptuales y de mayor flexibilidad en el análisis, el concepto multifractal no ha sido aplicado masivamente, posiblemente la complejidad de los parámetros multifractales constituye un impedimento para la interpretación de los resultados (Posadas et al., 2003). 
En el presente trabajo se utilizaron imágenes satelitales del tipo Lansat de cada año, desde 1987 al 2011, con resolución de 30 metros por pixel, y el método aplicado fue el de box counting, o método de la caja de contar, empleado para cuantificar los pixeles de la superficie glaciar y hallar posteriormente su volumen; asimismo se usó el Índice de Diferencia de Nieve Normalizada (NDSI) y el ratio imagen o relación de bandas $4 / 5$ para delimitar la superficie glaciar y diferenciar el hielo de otros elementos como nubes. A partir de la superficie obtenida, se estimó el volumen glaciar medio de la Cordillera Parón con la fórmula de Klein \& Isacks (1998).

Las imágenes satelitales escogidas fueron las del periodo de escasa precipitación y que no presentaban nubes sobre la superficie glaciar o en una mínima proporción que no representaban un problema para su digitalización.

El propósito de este artículo es presentar las bases del método multifractal y efectuar una aplicación de esta teoría para cuantificar el volumen y otros parámetros multifractales de los glaciares de la Cordillera Parón ante los eventos El Niño y La Niña.

\section{Materiales.}

Área de estudio.

El área de estudio abarca los glaciares de la Cordillera Parón (Artesonraju, Pirámide, Huandoy, Broggi, etc.), glaciares que circundan y abastecen a la laguna Parón, ubicada en la Cordillera Blanca de Perú, cerca a la ciudad de Caraz, y muy próxima al Huascarán (Figura 1).

La Cordillera Parón es una de las más elevadas de la Cordillera Blanca, dada su vecindad al glaciar más alto del Perú: El Huascarán (6 768 msnm). Sus coordenadas geográficas se hallan entre $-8^{\circ} 55^{\prime} 57^{\prime}$ ' a 9'3'43' de Latitud Sur, y Longitud Oeste entre 7743'23' a $-77^{\circ} 33$ '34' La Cordillera Blanca se ubica en la parte central y occidental del Perú, se extiende casi 180 kilómetros de norte a sur, tiene en total 663 glaciares, 269 lagunas y 41 ríos que son muy importantes para la hidroeléctrica del Cañón del Pato.

La ciudad de Caraz está tan solo a $20.25 \mathrm{~km}$ de

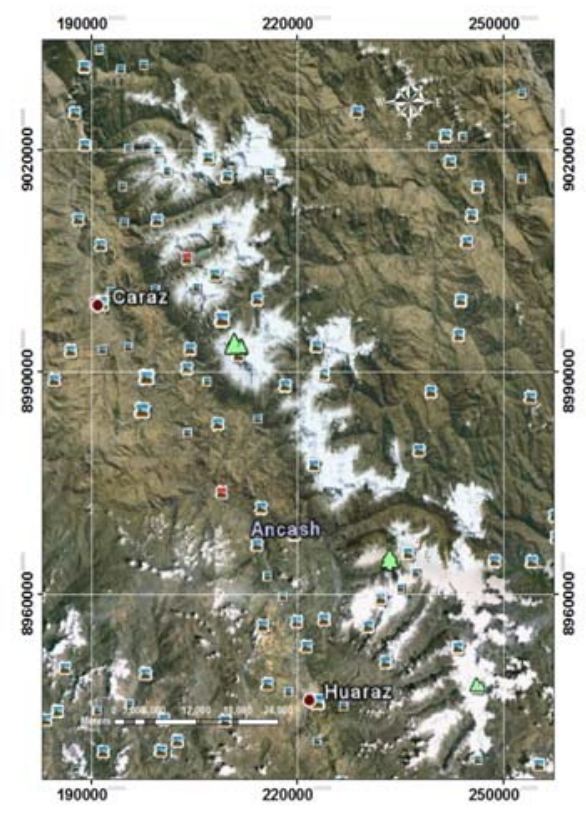

Figura 1. Posición geográfica de la Cordillera Parón dentro de la Cordillera Blanca Fuente: elaboración propia.

distancia de la Cordillera Parón; esta maqueta elaborada por la UGRH (Unidad de Glaciología y Recursos Hídricos) se hizo a escala 1/100000 (Figura 2).

Materiales.

Imágenes satelitales.- se usaron imágenes correspondientes al Mapeador Temático Landsat TM5, éste tiene 7 bandas, cada una capta una determinada longitud de onda, la resolución es de 30 metros por pixel; las imágenes Landsat son multiespectrales.

Se utilizaron imágenes de cada año desde 1987 al 2011 obtenidas del INPE (Instituto Nacional de Pesquisas Espaciais de Brasil) en la dirección http://www.inpe.br/.

Se eligieron imágenes del periodo seco o de escasa precipitación, y para diferenciar el agua o la nieve del hielo glaciar, se empleó el ratio imagen 4/5.

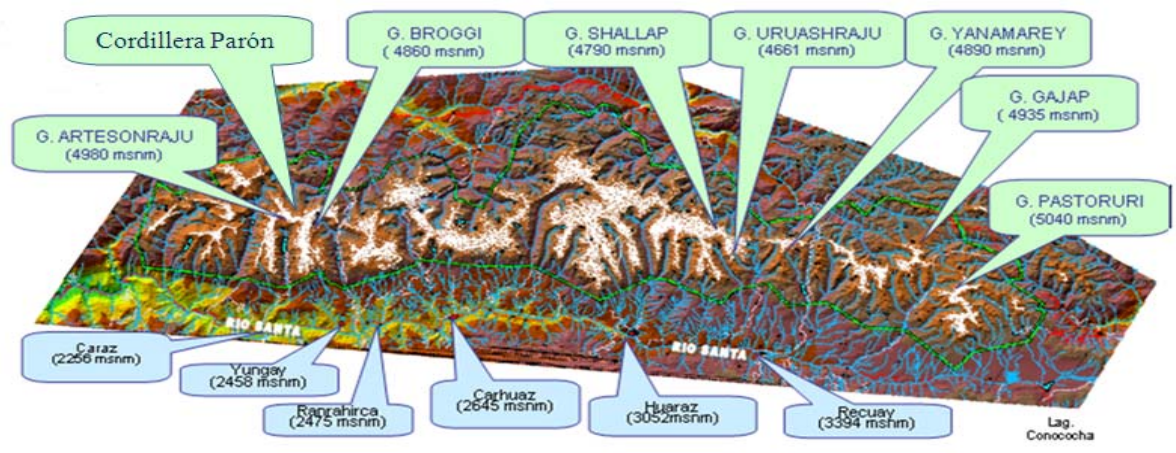

Figura 2. Ciudad de Caraz aledaña a la Cordillera Parón. Fuente: Unidad de Glaciología y Recursos Hídricos de la Autoridad Nacional del Agua. Escala: 1/100000. 
Tabla 1. Fuente de las imágenes de satélite Landsat TM5

\begin{tabular}{|c|c|c|c|c|c|}
\hline \multicolumn{6}{|c|}{ Cordillera Parón } \\
\hline Ruta & Fuente & Fecha & Ruta & Fuente & Fecha \\
\hline $8-66$ & INPE -Brasil & $31 / 05 / 1987$ & $8-66$ & INPE -Brasil & $21 / 07 / 2000$ \\
\hline $8-66$ & INPE-Brasil & 06/09/1988 & $8-66$ & INPE -Brasil & $05 / 05 / 2001$ \\
\hline $8-66$ & INPE-Brasil & 24/08/1989 & $8-66$ & INPE -Brasil & $01 / 08 / 2002$ \\
\hline $8-66$ & INPE -Brasil & $10 / 07 / 1990$ & $8-66$ & INPE -Brasil & $14 / 07 / 2003$ \\
\hline $8-66$ & INPE -Brasil & $15 / 09 / 1991$ & $8-66$ & INPE -Brasil & $29 / 05 / 2004$ \\
\hline $8-66$ & INPE-Brasil & $12 / 05 / 1992$ & $8-66$ & INPE -Brasil & $03 / 07 / 2005$ \\
\hline $8-66$ & INPE-Brasil & $31 / 05 / 1993$ & $8-66$ & INPE -Brasil & $06 / 07 / 2006$ \\
\hline $8-66$ & INPE -Brasil & $18 / 05 / 1994$ & $8-66$ & INPE -Brasil & $25 / 07 / 2007$ \\
\hline $8-66$ & INPE -Brasil & 09/08/1995 & $8-66$ & INPE -Brasil & $24 / 05 / 2008$ \\
\hline $8-66$ & INPE-Brasil & $11 / 08 / 1996$ & $8-66$ & INPE -Brasil & $30 / 07 / 2009$ \\
\hline $8-66$ & INPE -Brasil & $30 / 08 / 1997$ & $8-66$ & INPE -Brasil & $14 / 05 / 2010$ \\
\hline $8-66$ & INPE -Brasil & 02/09/1998 & $8-66$ & INPE -Brasil & $17 / 05 / 2011$ \\
\hline $8-66$ & INPE -Brasil & 20/08/1999 & & & \\
\hline
\end{tabular}

Como se aprecia en la Tabla 1, se utilizaron imágenes correspondientes a la estación seca (mayoseptiembre), momento en que el manto nival se encuentra en su mínimo anual, por tanto las imágenes escogidas no presentan problemas de precipitación que afecte el volumen glaciar, aún así se utilizó el ratio imagen $4 / 5$.

Las imágenes satelitales que se descargan de la página web, citadas anteriormente, vienen en 7 bandas o capas. En la Figura 3 se muestran 3 de ellas.

La Figura 3, muestra que las bandas 2 y 4 tienen valores de reflectancia parecida (la superficie glaciar tiene apariencia blanquesina), pero la banda 5 presenta mucha diferencia con las anteriores. Esto se puede corroborar con el perfil espectral hallado con el programa Erdas como se observa en la Figura 4.

En la Figura 4 (a), se muestra el glaciar y un punto negro donde se tomó el perfil espectral que se muestra en la Figura 4 (b).

Como se puede apreciar en la Figura 4 (b), las bandas 1 y 3 presentan mayor reflectancia (valor de pixel) y las bandas 5 y 7 tienen bajo valor de reflectancia.

Eventos el Niño y la Niña en el tiempo

La Tabla 2 muestra la ocurrencia de los fenómenos El Niño (ENSO) y La Niña hasta el año 2010, apreciándose que los eventos El Niño y La Niña son casi periódicos.
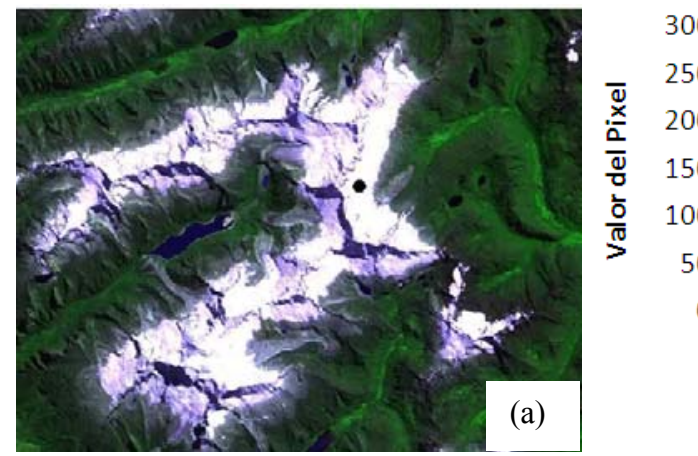

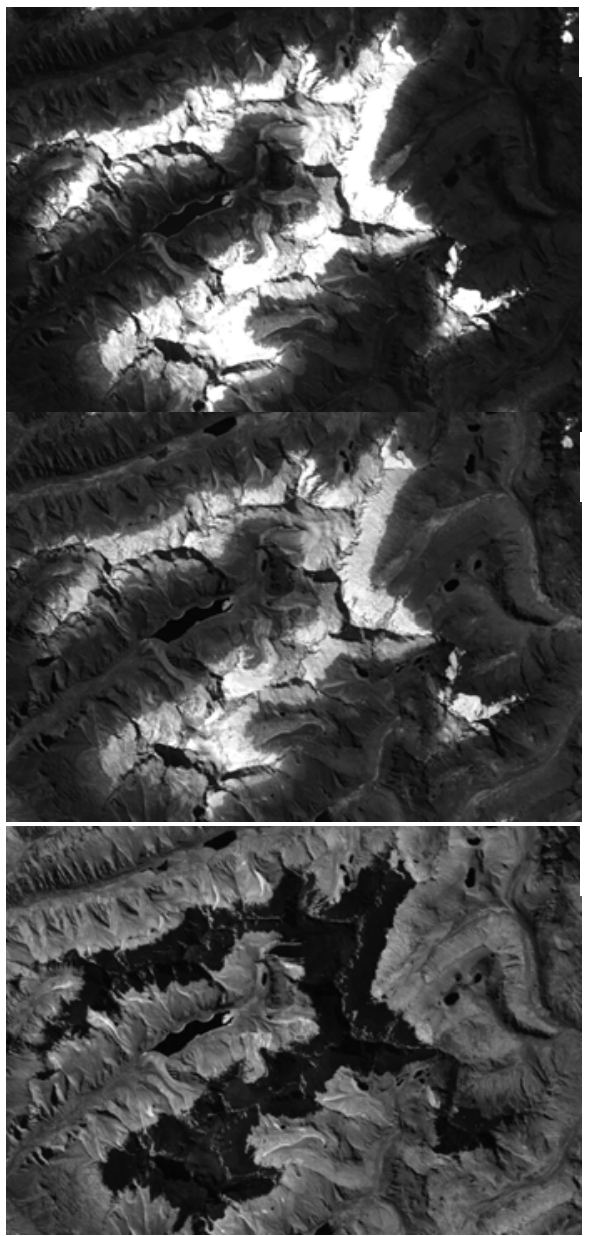

(a)

Figura 3. (a) banda 2, (b) banda 4 y (c) banda 5, respectivamente, de la Cordillera Parón del año 2011.

Los Niños más fuertes fueron los de 1982, 1991 y 1997, mientras que La Niña más fuertes fueron los de 1988, 2007 y 2010.

\section{Ecuaciones multifractales}

La probabilidad $P$ para sistemas no uniformes o heterogéneos se expresa como:

Figura 4. (a) Imagen combinada RGB 547 del 2011 de la Cordillera Parón, y (b) perfil espectral en un punto del glaciar. 
Tabla 2. Lista consensuada de El Niño y La Niña. Fuente: Jan Null, CCM disponible en http://ggweather.com/enso/oni.htm.

\begin{tabular}{cccccc}
\hline Invierno & WRCC & CDC & CPC & MEI & Consenso \\
\hline $1980-81$ & & & & & \\
$1981-82$ & & & & & \\
$1982-83$ & W+ & W & W+ & W+ & El Niño fuerte \\
$1983-84$ & & & C- & & \\
$1984-85$ & & & C- & C- & \\
$1985-86$ & & & & & \\
$1986-87$ & & & W & W & \\
$1987-88$ & W+ & W- & W & W- & El Niño \\
$1988-89$ & C+ & C- & C+ & C & La Niña fuerte \\
$1989-90$ & & & & & \\
$1990-91$ & & & W+ & & \\
$1991-92$ & W & W & W+ & W+ & El Niño fuerte \\
$1992-93$ & W & & W+ & W- & El Niño \\
$1993-94$ & W+ & & W & & \\
$1994-95$ & W+ & & W & W- & El Niño \\
$1995-96$ & & & C- & C- & \\
$1996-97$ & & & & & \\
$1997-98$ & W+ & W & W+ & W+ & El Niño fuerte \\
$1998-99$ & C+ & & C & C- & La Niña \\
$1999-00$ & & & C & C & \\
$2000-01$ & C & C & C- & C- & La Niña \\
$2001-02$ & & & & & \\
$2002-03$ & W & W & W & W & El Niño \\
$2003-04$ & & & & & \\
$2004-05$ & & W & & C+ & El Niño débil \\
$2005-06$ & & & & & \\
$2006-07$ & & W & & C+ & El Niño débil \\
$2007-08$ & W & & & C- & La Niña moder. \\
$2008-09$ & & & & & \\
$2009-10$ & & W & & C+ & El Niño fuerte \\
$2010-11$ & W & & & La Niña moder. \\
\hline
\end{tabular}

$$
P_{i}(\varepsilon) \sim \varepsilon^{\alpha_{i}}
$$

donde $\alpha_{i}$ es la fuerza de singularidad o exponente de Lipschitz-Hölder, $\varepsilon$ es la escala. Se puede caracterizar a los conjuntos multifractales sobre la base de las dimensiones generalizadas del momento de orden $q$ en una distribución, $D_{q}$ (Posadas et al., 2005):

$$
D_{q}=\lim _{\varepsilon \rightarrow 0}\left(\frac{1}{q-1} \frac{\log \mu(q, \varepsilon)}{\log (\varepsilon)}\right)_{(2)}
$$

La ecuación (2) se convierte en:

$$
D_{0}=\lim _{\varepsilon \rightarrow 0} \frac{\log (N(\varepsilon))}{\log (\varepsilon)}, D_{1}=\lim _{\varepsilon \rightarrow 0} \frac{\sum_{i=1}^{N(\varepsilon)} \mu_{i}(\varepsilon) \log \left(\mu_{i}(\varepsilon)\right)}{\log (\varepsilon)}, \quad \mathrm{D}_{2}=\lim _{\varepsilon \rightarrow 0} \frac{\log (C(\varepsilon))}{\log (\varepsilon)}
$$

Los valores $D_{0}, \quad D_{1}$ y $D_{2}$ son respectivamente la dimensión capacidad, la dimensión entropía y la dimensión correlación. $D_{0}$ proporciona información global acerca de un sistema. $D_{l}$ está relacionada a la información de la entropía; la dimensión correlación
$D_{2}$ calcula la correlación de medidas contenidas en una caja de tamaño $\varepsilon$ (Posadas et al., 2005). Debe cumplirse que:

$$
D_{2} \leq D_{1} \leq D_{0}
$$

La igualdad $D_{0}=D_{1}=D_{2}$ ocurre solamente si el fractal es estadísticamente o exactamente autosimilar y homogéneo. El espectro multifractal se calcula con:

$$
\begin{aligned}
& f(q)=-\lim _{N \rightarrow \infty} \frac{1}{\log (N)} \sum_{i=1}^{N(L)} \mu_{i}(q, L) \log \left[\mu_{i}(q, L)\right] \\
& \alpha(q)=-\lim _{N \rightarrow \infty} \frac{1}{\log (N)} \sum_{i=1}^{N(L)} \mu_{i}(q, L) \log \left[P_{i}(L)\right]
\end{aligned}
$$

El momento de orden $\mathrm{q}$ de la distribución estadística varió en pasos de 0.1 en 0.1

\section{Métodos.}

Diferenciación entre nieve y hielo en las imágenes remotas.

Dentro de la metodología del grupo de investigación, una de las mayores dificultades es diferenciar el hielo de la nieve en las imágenes de satélite, con el fin de analizar la evolución reciente de los glaciares de la Cordillera Parón y obtener una cartografía fiable, precisa y objetiva, se aplicó un análisis multitemporal a las imágenes; con la ayuda de los softwares Erdas 9.1, Envi 4.7, Image j y Arcgis 10 y de los criterios propuestos en la bibliografía (Dozier, 1981; Dozier, 1989).

En primer lugar se preparó la imagen en el software Erdas 9.1, especializado en tratamiento de imágenes de satélite, se combinó en imágenes conformadas por 3 bandas (RGB, 542). Después, con el programa Envi 4.7 se cortó la imagen combinada en un número redondo de pixeles, en nuestro caso fue de 700 x 500 pixeles. Nuevamente se lleva al Erdas donde separamos las bandas 5,4 y 2 y regresamos al Envi 4.7 para hallar, mediante una proporción matemática, una nueva capa raster NDSI (índice de nieve), y otra con el ratio imagen 4/5; estos dos últimos procesos nos permiten diferenciar la nieve del hielo en las imágenes.

a) NDSI (Indice Normalizado de Diferencia de Nieve).- El NDSI ayuda a distinguir la superficie glaciar de otras formas similares, como suelos brillantes, agua y nubes (Dozier, 1989). El NDSI se calcula con la siguiente relación:

$$
N D S I=(b 2-b 5) /(b 2+b 5)
$$

b) El ratio imagen 4/5.- es una simple razón entre las bandas 4 y 5 . La relación TM4/TM5 es citado por Jacobs et al (1997) como eficaz para discriminar el hielo de la nieve en estudios glaciológicos, especialmente a través de zonas de sombra.

$$
\text { Ratio imagen }=(b 4) /(b 5),(8)
$$




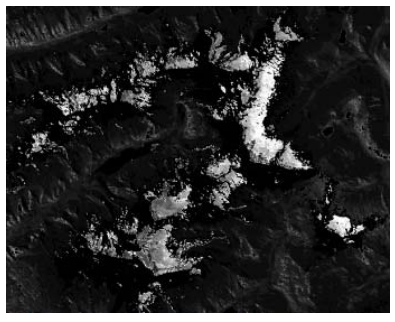

(a)

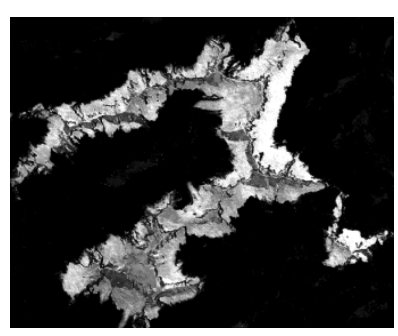

(b)

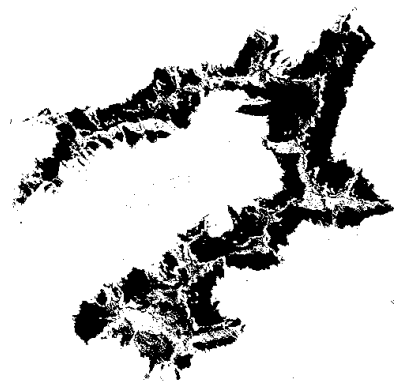

(c)

Figura 5. (a) Imagen NDSI, (b) Ratio 4/5, (c) Imagen binarizada, de la Cordillera Parón 2011.

El ratio imagen 4/5 también fue utilizado por Posadas et al. (2005) como eficaz para diferenciar la masa de ulexite de otros materiales que no lo son.

Determinación de la superficie y volumen glaciar.

a. Con Sistema de Información Geográfica.- las imágenes obtenidas a partir del NDSI y el ratio imagen se binarizaron con el programa Image $\mathrm{j}$; correspondiendo un valor 1 para hielo y cero para otros elementos. La imagen binarizada se llevó al Arc Gis para hallar el área.

b. Con Multifractales.- La superficie glaciar se halló con la ecuación:

$$
P_{i}(L) \approx L^{\alpha_{i}}
$$

donde $\mathrm{P}_{\mathrm{i}}$ es la probabilidad de hallar cierta cantidad de "materia", L es el tamaño variable de cuadrícula y $\alpha$ es el exponente de Holder; hay que tener en cuenta que 1 pixel equivale a 30 metros, por lo tanto al resultado lo multiplicamos por 900 .

Obtenida la superficie glaciar, por cualquiera de los métodos a ó b, se estimó el volumen medio del glaciar con la siguiente fórmula (Klein \& Isacks, 1998):

$$
V=C \cdot A^{b}(10)
$$
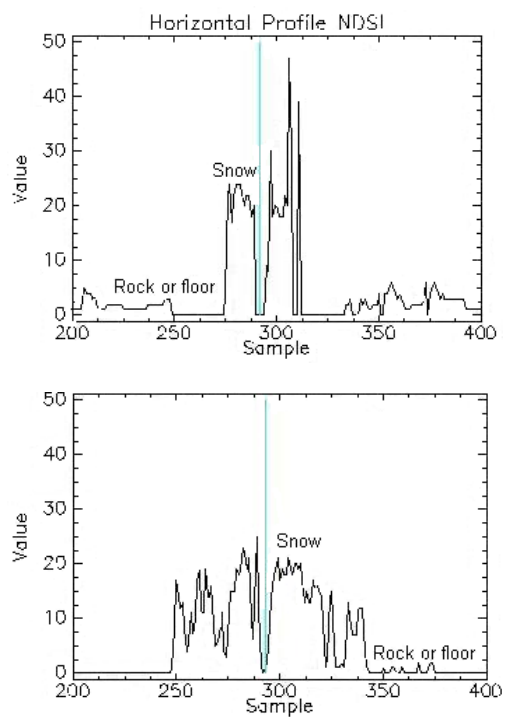

Figura 6. (a) Perfil horizontal NDSI 2011. (b) Perfil horizontal para el ratio $4 / 5$.

donde, $\mathrm{V}$ es el volumen glaciar en $\mathrm{km}^{3}$, A es la superficie glaciar en $\mathrm{km}^{2}, \quad \mathrm{C}=0,048$ (valor empírico) y $\mathrm{b}=1,36$ (valor empírico).

Determinación de los parámetros multifractales

La imagen binarizada con el programa Image $\mathrm{j}$, se grabó en formato texto y se procesó con el software Multifractal Analysis \& Scalling System (MASS) y puede ser obtenido de http://inrm.cip.cgiar.org/home/downmod.htm (software elaborado por el Centro Internacional de la Papa) especial para multifractales e Ingeniería, con él

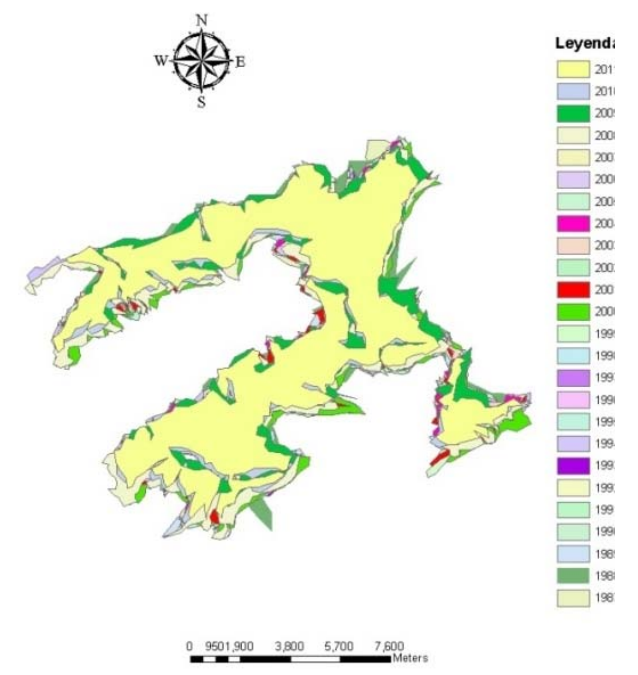

Figura 7. Deglaciación de la Cordillera Parón en el tiempo.

se aplicó el método de box counting, que consiste en 


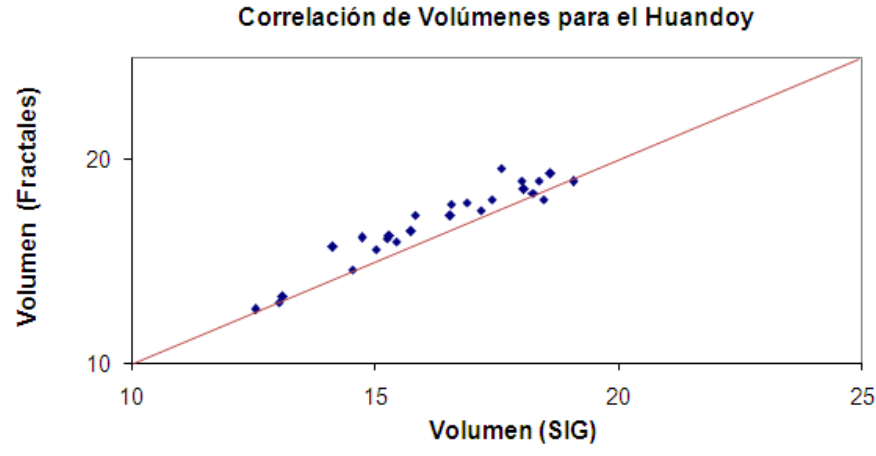

Figura 8. Correlación entre los volúmenes obtenidos por SIG y por Multifractales.

contabilizar en cada celda la cantidad de "materia glaciar". En el programa Mass se buscó la linealidad y se buscó un modelo que tiene un coeficiente de determinación del 98 al 99\%. Luego, se editó la tabla, la cual nos reportó las dimensiones $D_{0}, D_{1}$ y $D_{2}, \mathrm{y}$ después se exportaron los datos al Excel.

Comparación entre el NDSI y el ratio imagen $4 / 5$

El índice de nieve (NDSI) y el ratio imagen $4 / 5$ se obtuvieron a partir de las bandas 2, 4 y 5 empleando las ecuaciones (7) y (8), respectivamente.

Los Perfiles Horizontales de la Cordillera Parón fueron obtenidos con Erdas y se muestran en la Figura 6.

Tomando en cuenta las Figuras 5 y 6 , el método del ratio imagen $4 / 5$ resultó mejor que el NDSI para discriminar o separar sombras de nubes, esto es corroborado por Vargas et al., (2009); en el caso de las áreas con suelo y/o roca tienen valores más uniformes con respecto a la cobertura glaciar. Por lo tanto, la imagen obtenida con el ratio 4/5, se binarizó (Figura 5c) $\mathrm{y}$ en formato texto fue analizada con el software MASS.

Resultados y discusión.

Cuantificación del Volumen Glaciar.

El volumen glaciar de la Cordillera Parón ha venido decreciendo en el tiempo, tal como se aprecia a continuación.

En la Figura 7 se aprecia que en los años en que se presentó el evento La Niña (1988, 2000 y 2011), los volúmenes son más grandes que cuando El Niño (1987, 1991 y 1997).

La cuantificación del volumen glaciar de la cordillera Parón, obtenida tanto con el método convencional o SIG y por medio de los multifractales, presentado en la Tabla 3, muestra que la Cordillera Parón perdió un promedio de $18 \%$ de su volumen glaciar en 24 años, esto es corroborado por PNUMA (2007).

Correlación de volúmenes con SIG y Multifractales.
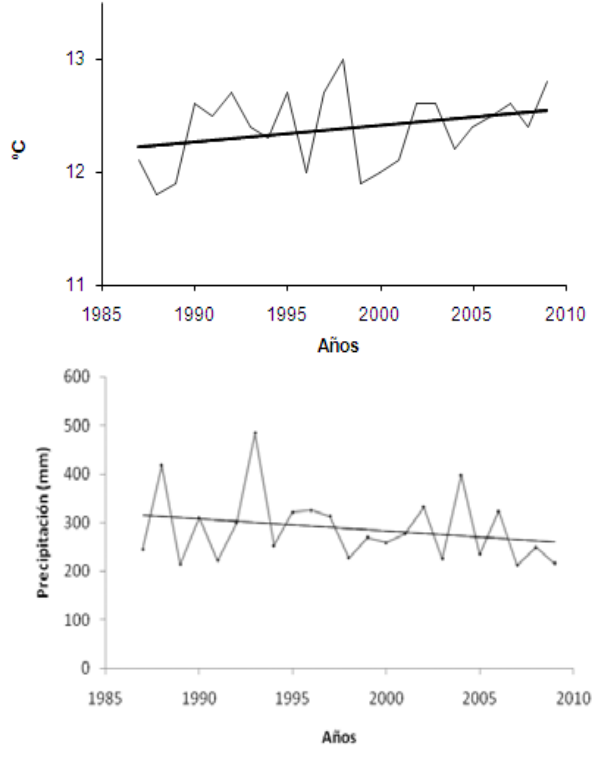

Figura 9. (a) Tendencia de la temperatura y (b) Tendencia de la precipitación histórica, registradas en estaciones cercanas a la Cordillera Parón.

Tabla 3.Variación del volumen glaciar de la Cordillera Parón

\begin{tabular}{|c|c|c|c|c|c|c|c|}
\hline \multicolumn{8}{|c|}{ Cordillera Parón } \\
\hline & \multicolumn{2}{|c|}{ SIG } & & \multicolumn{4}{|c|}{ Multifractales } \\
\hline Año & $\begin{array}{c}\mathrm{A} \\
\left(\mathrm{km}^{2}\right)\end{array}$ & $\begin{array}{c}\mathrm{V} \\
\left(\mathrm{km}^{3}\right)\end{array}$ & $\%$ & Año & $\begin{array}{c}\mathrm{A} \\
\left(\mathrm{km}^{2}\right)\end{array}$ & $\begin{array}{c}\mathrm{V} \\
\left(\mathrm{km}^{3}\right)\end{array}$ & $\%$ \\
\hline 1987 & 70.77 & 15.74 & 100 & 1987 & 73.16 & 16.47 & 100 \\
\hline 1988 & 81.45 & 19.06 & 121 & 1988 & 81 & 18.91 & 115 \\
\hline 1989 & 79.55 & 18.45 & 117 & 1989 & 78.31 & 18.06 & 110 \\
\hline 1990 & 67.41 & 14.73 & 94 & 1990 & 72.16 & 16.16 & 98 \\
\hline 1991 & 65.39 & 14.14 & 90 & 1991 & 70.64 & 15.70 & 95 \\
\hline 1992 & 78.91 & 18.25 & 116 & 1992 & 79.28 & 18.37 & 112 \\
\hline 1993 & 73.5 & 16.57 & 105 & 1993 & 77.49 & 17.81 & 108 \\
\hline 1994 & 76.18 & 17.40 & 111 & 1994 & 78.25 & 18.05 & 110 \\
\hline 1995 & 71.03 & 15.82 & 100 & 1995 & 75.79 & 17.28 & 105 \\
\hline 1996 & 74.54 & 16.89 & 107 & 1996 & 77.72 & 17.88 & 109 \\
\hline 1997 & 66.76 & 14.54 & 92 & 1997 & 67.01 & 14.61 & 89 \\
\hline 1998 & 79.28 & 18.37 & 117 & 1998 & 81.21 & 18.98 & 115 \\
\hline 1999 & 73.41 & 16.55 & 105 & 1999 & 75.67 & 17.24 & 105 \\
\hline 2000 & 75.48 & 17.18 & 109 & 2000 & 76.51 & 17.50 & 106 \\
\hline 2001 & 78.12 & 18.01 & 114 & 2001 & 81.08 & 18.94 & 115 \\
\hline 2002 & 68.41 & 15.03 & 95 & 2002 & 70.28 & 15.59 & 95 \\
\hline 2003 & 78.3 & 18.06 & 115 & 2003 & 79.8 & 18.53 & 113 \\
\hline 2004 & 69.14 & 15.25 & 97 & 2004 & 71.98 & 16.11 & 98 \\
\hline 2005 & 69.26 & 15.29 & 97 & 2005 & 72.42 & 16.24 & 99 \\
\hline 2006 & 80 & 18.60 & 118 & 2006 & 82.25 & 19.31 & 117 \\
\hline 2007 & 69.82 & 15.45 & 98 & 2007 & 71.58 & 15.99 & 97 \\
\hline 2008 & 76.79 & 17.59 & 112 & 2008 & 83.03 & 19.56 & 119 \\
\hline 2009 & 61.62 & 13.04 & 83 & 2009 & 61.37 & 12.97 & 79 \\
\hline 2010 & 59.89 & 12.54 & 80 & 2010 & 60.21 & 12.64 & 77 \\
\hline 2011 & 61.85 & 13.11 & 83 & 2011 & 62.38 & 13.26 & 81 \\
\hline Prom & 72.27 & 16.23 & & Prom & 74.42 & 16.89 & \\
\hline Desv.est & 6.24 & 1.89 & & Desv.est & 6.48 & 1.97 & \\
\hline
\end{tabular}



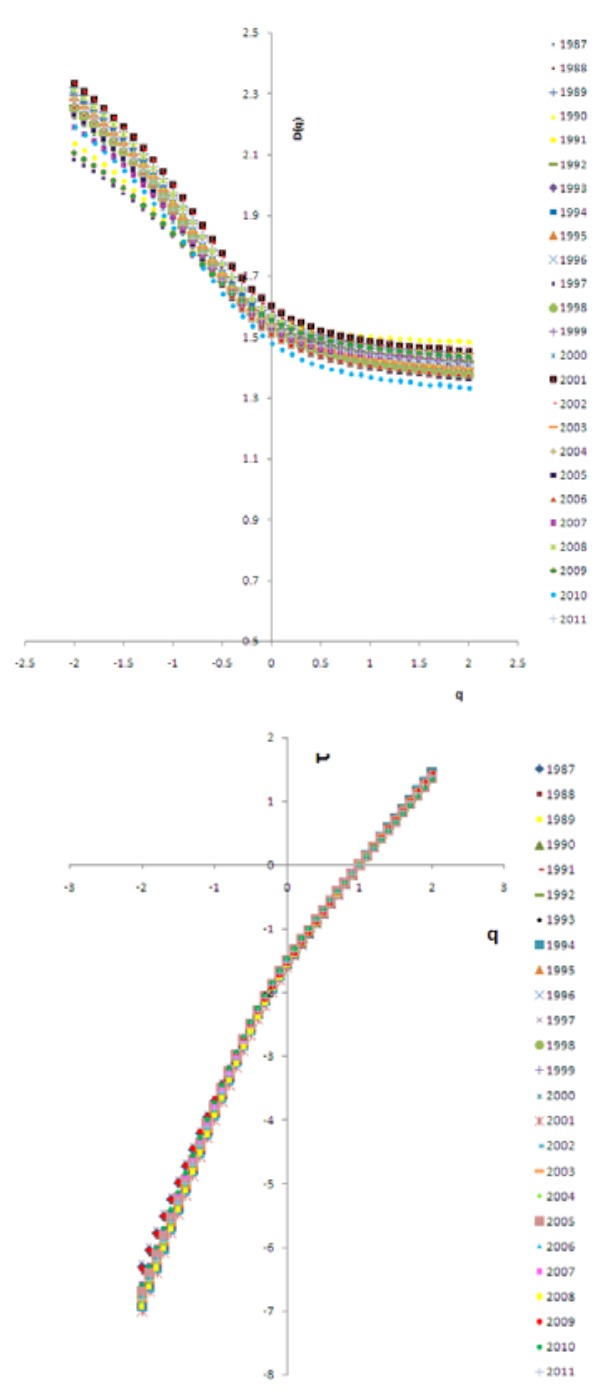

Figura 10. (a) Función $q$ vs $D q$ y (b) función $q$ vs $\tau$, obtenidos con multifractales.

La dispersión entre los volúmenes obtenidos con SIG y con Multifractales, se presenta en la Figura 8.

El coeficiente de correlación de Pearson resultó cercano a uno $(\mathrm{r}=0.957)$, y la prueba de $\mathrm{T}$ como muestras relacionadas resultó no significativo $(\mathrm{p}=$ 0.63); al no encontrarse diferencias significativas se puede asumir que es equivalente el uso de ambas metodologías para determinar el volumen glaciar.

Tendencia de la temperatura y precipitación en la zona.

La temperatura y precipitación en el entorno

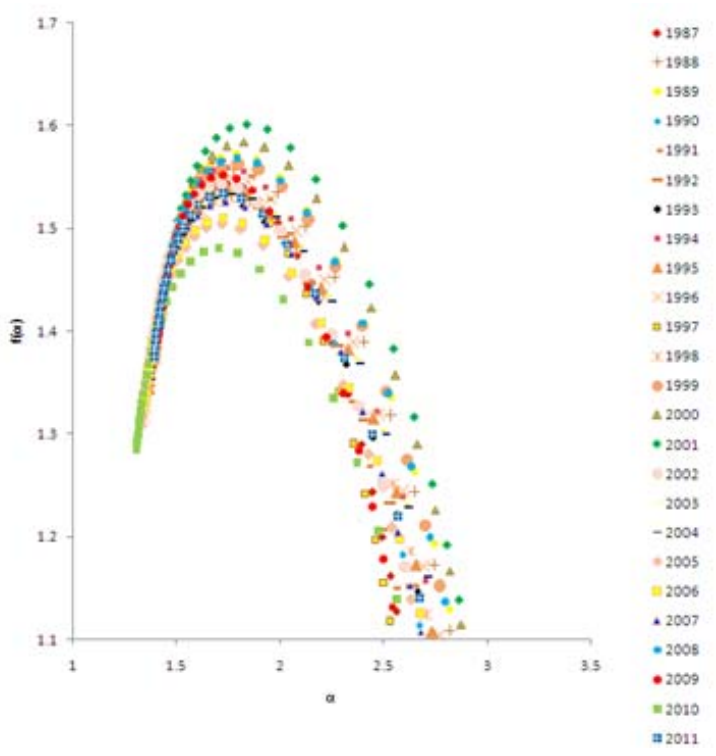

Figura 11. Espectros multifractales de la Cordillera Parón desde 1987 al 2011.

glaciar se comportó como se muestra en la Figura 9.

La Figura 9, muestra una tendencia creciente de la temperatura media anual de alrededor de $0.35{ }^{\circ} \mathrm{C}$, mientras que la precipitación viene disminuyendo, lo que es corroborado por Bates et al. (2008).

Función q vs Dq y q vs $\tau$.

Los resultados de la función multifractal $(D q)$ y el exponente de masa $(\tau)$ se muestran en la Figura 10.

La función multifractal $(D q)$ y la función $\tau$ (exponente de masa), tienen un quiebre en $q=0$, es decir las curvas tienen dos pendientes diferentes. Este quiebre es atribuible a que existe heterogeneidad dentro del sistema, siendo su comportamiento bifractal o en general multifractal.

Comportamiento multifractal.

El espectro multifractal obtenido con el software Mass, tal como se muestra en la Figura 11, presenta una abertura del espectro multifractal $(\Delta \alpha)$ variable, dándonos una idea de la dispersión de la información.

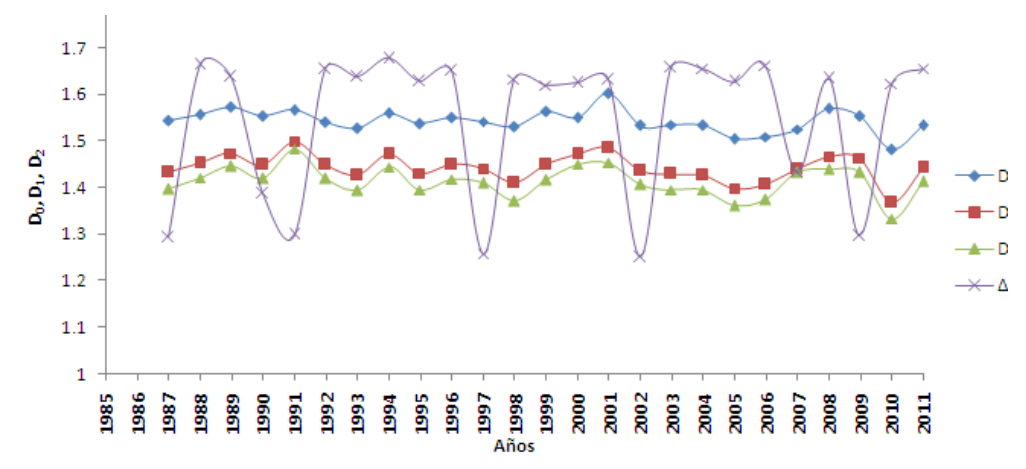

Figura 12. Variación de las dimensiones fractales $D_{0}, D_{1}, D_{2}$ y $\Delta \alpha$, para el glaciar de la Cordillera Parón. 
Parámetros Multifractales.

La dimensión capacidad $\left(\boldsymbol{D}_{0}\right)$, la dimensión entropía $\left(\boldsymbol{D}_{1}\right)$, la dimensión correlación $\left(\boldsymbol{D}_{2}\right)$, y el tamaño de abertura del espectro $(\Delta \boldsymbol{\alpha})$ se muestran en la Tabla 4.

Por lo tanto la dimensión fractal $D_{0}$ promedio es de $1.54, D_{1}=1.44$ y $D_{2}=1.41$.

Comparando los años con la dimensión capacidad $\left(\boldsymbol{D}_{0}\right)$, la dimensión entropía $\left(\boldsymbol{D}_{1}\right)$, la dimensión correlación $\left(\boldsymbol{D}_{2}\right)$, y la abertura $(\Delta \boldsymbol{\alpha})$ se obtiene la figura 12.

El parámetro multifractal $\Delta \alpha$ es el que mejor reflejó la presencia de los eventos El Niño con puntos más bajos (1987, 1991, 1997, 2002, y 2009) y puntos más elevados cuando se presentó los eventos la Niña (1988, 1998, 2000, y 2007).

Tabla 4. Parámetros Multifractales del glaciar de la Cordillera Parón

\begin{tabular}{lllll}
\hline AÑO & $D_{0}$ & $D_{1}$ & $D_{2}$ & $\Delta \alpha$ \\
\hline $1987 \mathrm{EN}$ & 1.543 & 1.433 & 1.396 & 1.293 \\
$1988 \mathrm{LN}$ & 1.557 & 1.453 & 1.421 & 1.664 \\
1989 & 1.573 & 1.473 & 1.446 & 1.637 \\
1990 & 1.553 & 1.448 & 1.419 & 1.387 \\
$1991 \mathrm{EN}$ & 1.566 & 1.499 & 1.483 & 1.298 \\
1992 & 1.539 & 1.448 & 1.419 & 1.654 \\
1993 & 1.527 & 1.426 & 1.393 & 1.637 \\
1994 & 1.559 & 1.472 & 1.444 & 1.677 \\
1995 & 1.537 & 1.431 & 1.395 & 1.627 \\
1996 & 1.549 & 1.449 & 1.418 & 1.652 \\
$1997 \mathrm{EN}$ & 1.541 & 1.441 & 1.409 & 1.258 \\
$1998 \mathrm{LN}$ & 1.529 & 1.410 & 1.371 & 1.633 \\
1999 & 1.562 & 1.449 & 1.415 & 1.619 \\
2000 & 1.550 & 1.471 & 1.450 & 1.624 \\
2001 & 1.601 & 1.486 & 1.453 & 1.632 \\
2002 & 1.532 & 1.437 & 1.407 & 1.250 \\
2003 & 1.535 & 1.429 & 1.395 & 1.657 \\
$2004 \mathrm{EN}$ & 1.533 & 1.426 & 1.395 & 1.655 \\
2005 & 1.505 & 1.398 & 1.362 & 1.627 \\
2006 & 1.509 & 1.406 & 1.373 & 1.661 \\
$2007 \mathrm{LN}$ & 1.525 & 1.439 & 1.431 & 1.432 \\
2008 & 1.569 & 1.466 & 1.439 & 1.633 \\
$2009 \mathrm{EN}$ & 1.552 & 1.463 & 1.433 & 1.297 \\
2010 & 1.481 & 1.368 & 1.333 & 1.623 \\
2011 & 1.528 & 1.399 & 1.366 & 2.036 \\
Promedio & 1.542 & 1.441 & 1.411 & 1.567 \\
\hline & & & &
\end{tabular}

\section{Conclusiones.}

1.- El volumen glaciar tuvo un comportamiento variable en el tiempo; la reducción promedio del volumen glaciar de la Cordillera Parón obtenido por SIG y Multifractales fue del $18 \%$ en el periodo 1987 al 2011.
2.- No se encontraron diferencias estadísticamente significativas entre los volúmenes hallados con métodos SIG o multifractales.

3.- El parámetro multifractal $\Delta \alpha$ es el que mejor revela la presencia de los eventos El Niño y La Niña. El promedio de la dimensión entropía $D_{l}$ fue de 1.44

\section{Literatura citada.}

Bates B., Kundzevicz S. \& Palutikof J. 2008. Climate change and water. Tech- nical Paper of the Intergovernmental Panel on climate Change, IPCC.Se cretariat, Geneva $210 \mathrm{pp}$.

Dozier J. 1989. Spectral signature of alpine snow cover from the Landsat Thema-tic Mapper. Remote sensing of environment, 28: 9-22.

Jacobs J., Simms E. \& Simm A. 1997. Recession of the southern part of Barnes Ice Cap, Baffin Island, Canada, between 1961 and 1993, determined from digital mapping of Lansat TM. Journal of Glaciology, 43, 98 102.

Klein A. \& Isacks B. 1998. Alpine glacial geomorphological studies in the central Andes using Landsat thematic mapper images. Glacial Geology and Geomorphology; rp01/1998.

Medina G. \& Mejía A. 2010. Incidencia de los eventos El Niño y La Niña en el comportamiento de glaciares tropicales en Perú. Revista ECI Perú,

Montero E. 2003. Aplicación de técnicas de análisis multifractal a distribuciones de tamaño-volumen de partículas de suelo obtenidas mediante análisis por difracción de láser. Tesis Doctoral, Universidad Politécnica de Madrid. $212 \mathrm{p}$

PNUMA. 2007. ¿El fin de las cumbres nevadas? Glaciares y Cambio Climático en la Comunidad Andina. Secretaría General de la Comunidad Andina, PN-UD para el Medio Ambiente, $104 \mathrm{p}$.

Posadas A., Gimenez D. \& Quiroz R. 2002. Análisis Multifractal de la variabilidad espacial de la conductividad hidráulica en un suelo estratificado. Revista de Investigación de Física ISSN16057744.Vol.5 (36.43).

, Gimenez D., Quiroz R. \& Protz R. 2003. Multifractal characterization of soil pore systems. Soil Science Society of America Journal, 67, pp. 1361-1369. ., Quiroz R., Zorogastúa R. \& Leon C. 2005. Multifractal characterization of the spatial distribution of ulexite in a Bolivian salt flat. International Journal of Remote Sensing. Month 2005, 1-13.

Urrutia R. \& Vuille M. 2009. Climate change projections for the tropical Andes using a regional climate model: Temperature and precipitation simulations. Journal of Geophysical Research, vol. 114.

Vargas C., Villon C. \& Pasapera J. 2009. Comparación de Técnicas para el Mapeo de Cobertura Glaciar con Imágenes LANDSAT y ASTER en la Cordillera Blanca. Simposio Brasileiro de Sensoramiento Remoto, Natal, Brasil, INPE, p. 6911-6917.

\footnotetext{
${ }^{1}$ Universidad Nacional Agraria de la Selva, Casilla 156 Tingo María, Av. Universitaria km 1.5, 2011, gmeddiaz@gmail.com, telef: (062)564041.

2 Universidad Nacional Agraria La Molina. Av. La Molina, La Molina, Lima 12, Perú, 2011, jabel@lamolina.edu.pe, telef: 3401180, telefax: 3499991.
} 